04,07, 19

\title{
Атомистическое моделирование сегнетоэлектрика-сегнетоэластика молибдата гадолиния
}

\author{
(C) В.Б. Дудникова ${ }^{1}$, Е.В. Жариков ${ }^{2}$ \\ ${ }^{1}$ Московский государственный университет им. М.В. Ломоносова, \\ Москва, Россия \\ ${ }^{2}$ Институт общей фризики им. А.М. Прохорова РАН, \\ Москва, Россия \\ E-mail: VDudnikova@hotmail.com \\ (Поступила в Редакцию 18 мая 2016 г. \\ В окончательной редакции 20 августа 2016 г.)
}

\begin{abstract}
Методом межатомных потенциалов проведено моделирование ромбического сегнетоэлектрика-сегнетоэластика $\left(\beta^{\prime}\right.$-фазы) молибдата гадолиния $\mathrm{Gd}_{2}\left(\mathrm{MoO}_{4}\right)_{3}$. Для моделирования использовалась программа GULP 4.0.1 (General Utility Lattice Program), в основу которой положена процедура минимизации энергии кристаллической структуры.

Параметры потенциалов межатомного взаимодействия гадолиний-кислород определены подгонкой к экспериментальным структурным данным и упругим константам в рамках процедуры, предусмотренной в программе GULP. Атомистическое моделирование с использованием эффективных зарядов атомов и полученной системы межатомных потенциалов позволило сделать разумные оценки структурных параметров и координат атомов, важнейших физических, механических и термодинамических свойств этих кристаллов. Получены температурные зависимости теплоемкости кристалла и колебательной энтропии. Рассчитанные значения параметров потенциалов взаимодействия гадолиний-кислород могут быть использованы для моделирования более сложных гадолиний-содержащих соединений.
\end{abstract}

Работа выполнена при финансовой поддержке РФФИ, проект № 15-05-06742.

DOI: 10.21883/FTT.2017.05.44369.196

\section{1. Введение}

Значительный интерес к молибдату гадолиния, $\mathrm{Gd}_{2}\left(\mathrm{MoO}_{4}\right)_{3}$ (GMO) появился после публикации Борхардта и Берштедта [1], сообщивших в 1966 г. о его сегнетоэлектрических свойствах.

GMO претерпевает сложные полиморфные превращения в диапазоне температур от температуры плавления до комнатной $[2,3]$. Известны $\alpha$-, $\beta$ - и $\beta^{\prime}$-фазы. Есть также упоминание о существовании $\gamma$-фазы [3] вблизи температуры плавления, которая составляет $1160^{\circ} \mathrm{C}$ [4].

При высоких температурах (выше $850^{\circ} \mathrm{C}$ ) устойчива высокотемпературная тетрагональная параэлектрическая $\beta$-фаза. Ниже $850^{\circ} \mathrm{C}$ устойчивой становится моноклинная $\alpha$-фаза. Однако переход $\beta$-фазы в $\alpha$-фазу затруднен. Для его осуществления требуется продолжительная выдержка при температуре фазового перехода $\left(850^{\circ} \mathrm{C}\right)$ [4]. В обычных условиях роста кристалла из расплава при охлаждении ниже $850^{\circ} \mathrm{C}$ переход в стабильную $\alpha$-фазу не происходит, и кристалл находится в метастабильном состоянии $\beta$-фазы. При дальнейшем охлаждении по достижении температуры Кюри, $159^{\circ} \mathrm{C}$, $\beta$-фаза переходит в низкотемпературную сегнетоэлектрическую и сегнетоэластическую ромбическую $\beta^{\prime}$-фазу. При кристаллизации из расплава методом Чохральского могут быть получены кристаллы $\beta^{\prime}$-GMO высокого оптического качества $[4,5]$.
В зависимости от условий синтеза при комнатной температуре могут существовать как стабильная $\alpha$-фаза, так и метастабильная $\beta^{\prime}$-фаза. Хотя ромбическая $\beta^{\prime}$-модификация термодинамически метастабильна [6], однако кинетически она очень устойчива и в условиях окружающей среды может годами сохранять свое состояние, не переходя в низкотемпературную моноклинную $\alpha$-фазу [7]. Кристаллическая структура $\beta^{\prime}$-GMO изучалась в целом ряде работ [1-3,8-13]. Сегнетоэлектрическая и сегнетоэластическая фаза $\mathrm{Gd}_{2}\left(\mathrm{MoO}_{4}\right)_{3}$ ( $\beta^{\prime}$-фаза) имеет ромбическую симметрию и относится к пространственной группе Pba2 с четырьмя формульными единицами в элементарной ячейке. Атомы гадолиния окружены семью атомами кислорода, атомы молибдена четырьмя. Полиэдры $\mathrm{GdO}_{7}$ соединены углами с молибденовыми тетраэдрами (рис. 1). Результаты детального исследования структуры $\beta^{\prime}$-GMO опубликованы почти одновременно в независимых работах [3] и [8,9]. Хотя параметры элементарной ячейки, по данным этих работ, имеют близкие значения, атомные координаты различаются. Возможной причиной этих различий является наличие двойникования в кристаллах, исследованных в работе [3]. Дальнейшие исследования позволили установить ряд важных особенностей структуры GMO, в частности, были обнаружены новый структурный переход при $200 \mathrm{~K}$ [10], пять структурных переходов при повышенных давлениях и исследована роль структурного разупорядочения в аморфизации GMO [11]. Детально 


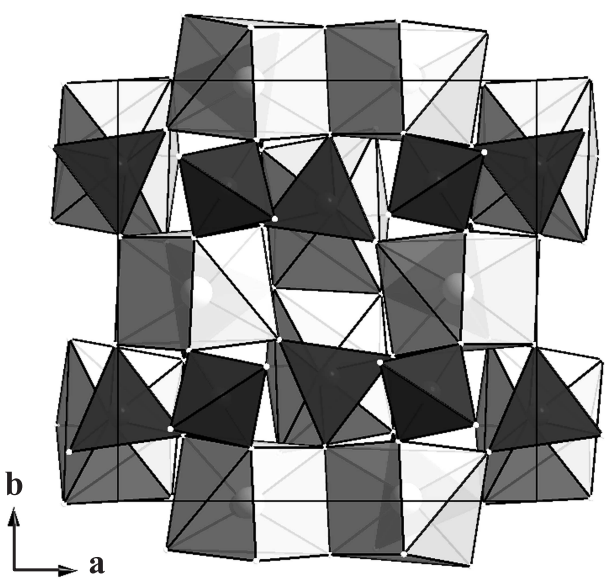

Рис. 1. Структура $\beta^{\prime}$-фазы $\mathrm{Gd}_{2}\left(\mathrm{MoO}_{4}\right)_{3}$.

изучена трансформация $\alpha$-фазы в $\beta^{\prime}$-фазу, характеризующаяся значительным $(\sim 24 \%)$ изменением объема элементарной ячейки [12]. Новая методология, предложенная в работе [13], дала возможность получить более детальное описание разупорядоченной структуры GMO, в том числе выполнить систематическую характеризацию теплового поведения этого кристалла.

Кристалл $\beta^{\prime}$-GMO является наиболее интересной полиморфной модификацией молибдата гадолиния. Он принадлежит к классу несобственных („неправильных“) сегнетоэлектриков $[14,15]$. В сегнетоэлектрическом состоянии он обладает также сегнетоэластическими свойствами. Крупные кристаллы $\beta^{\prime}$-GMO могут быть выращены непосредственно из расплава, они прозрачны и бесцветны. Это позволяет использовать их в различных устройствах оптоэлектроники и иных приложениях.

Молибдат гадолиния представляет значительный интерес в качестве матрицы для создания твердотельных лазеров, излучающих в различных областях спектра [16-19], люминофоров [20,21] и светодиодов белого цвета $[22,23]$. Активно продолжают изучаться магнитные, нелинейно-оптические свойства $\beta^{\prime}$-GMO [24-27].

$\mathrm{B}$ отличие от других полиморфных модификаций, структурные и физические характеристики $\beta^{\prime}$-GMO изучены в достаточной степени (см. обзоры $[28,29]$ ). Это расширяет возможности успешного моделирования. Вместе с тем наличие сегнетоэластических свойств, доменной структуры и других особенностей этого кристалла вызывает вопрос о том, можно ли его адекватно описать с помощью простой модели межатомных потенциалов, подобной использованной ранее в работе [30], или требуется существенное усложнение модели и введение специфических потенциалов взаимодействия.

Настоящая работа посвящена моделированию структуры, механических и термодинамических свойств $\beta^{\prime}$-GMO. В задачи работы входила также отработка на молибдате гадолиния методики моделирования и создания системы межатомных потенциалов, пригодных для дальнейшего использования при моделировании родственных данному соединению, но более сложных двойных молибдатов и вольфраматов, являющихся перспективными средами твердотельных лазеров.

\section{2. Методика моделирования}

Моделирование было проведено методом межатомных потенциалов с помощью программы GULP 4.0.1 (General Utility Lattice Program) [31], в основу которой положена процедура минимизации энергии кристаллической структуры.

Атомистический подход основан на использовании эмпирически определенных межатомных потенциалов, которые описывают взаимодействие между ионами в кристалле. Парный потенциал $U_{i j}$ взаимодействия ионов $i$ и $j$ с зарядами $q_{i}$ и $q_{j}$ является алгебраической суммой нескольких составляющих

$$
U_{i j}\left(R_{i j}\right)=q_{i} q_{j} e^{2} / R_{i j}+A_{i j} \exp \left(-R_{i j} / \rho_{i j}\right)-C_{i j} / R_{i j}^{6}
$$

Первый член учитывает кулоновское взаимодействие, второй - отталкивание, возникающее при перекрывании электронных оболочек соседних атомов, а последний учитывает ван-дер-ваальсово взаимодействие. Здесь $R_{i j}$ - межатомное расстояние, $A_{i j}, \rho_{i j}, C_{i j}-$ эмпирические параметры короткодействующих потенциалов, область действия которых в настоящей работе составляла $15 \AA$ для связи кислород-кислород и $10 \AA$ в остальных случаях. Эффективным способом учета ковалентной составляющей является учет поляризуемости ионов, чаще всего анионов. В наших вычислениях поляризуемость кислородного иона учитывалась с помощью „оболочечной модели“ [32]. В этом подходе ионы описываются как точечные положительно заряженные ядра, содержащие всю массу и окруженные отрицательно заряженной оболочкой, с помощью которой моделируется валентное электронное облако. Остов и оболочка связаны гармонической упругой константой $\chi_{i}$

$$
U_{i}^{s}=(1 / 2) \chi_{i} l_{i}^{2},
$$

где $l_{i}$ - расстояние между центрами остова и смещенной оболочки.

Для моделирования структурных, механических и термодинамических свойств кристаллов необходим набор согласованных межатомных потенциалов. В атомистическом моделировании довольно часто и успешно используется принцип трансферабельности межатомных потенциалов. Согласно этому принципу, набор параметров потенциалов, определенный для относительно простой и хорошо изученной системы, может быть перенесен на некоторую группу соединений, включая более сложные системы, со сходным характером химических связей (см., например [33,34]). Подходящая согласованная система межатомных потенциалов была получена в работе [35] подгонкой к структурным и 
Таблица 1. Параметры потенциалов межатомных взаимодействий и заряды атомов, использованные в работе; $\mathrm{O}_{C}-$ остов иона кислорода, $\mathrm{O}_{S}$ - валентная оболочка иона кислорода

\begin{tabular}{c|c|c|c|c|c}
\hline \multirow{2}{*}{$\begin{array}{c}\text { Взаимо- } \\
\text { действие }\end{array}$} & \multicolumn{2}{|c|}{ Параметры потенциалов } & \multirow{2}{*}{ Атом } & \multirow{2}{*}{ Заряд, $e$} \\
\cline { 2 - 4 } & $A, \mathrm{eV}$ & $\rho, \AA$ & $c, \mathrm{eV} \AA^{6}$ & & \\
\hline $\mathrm{Gd}-\mathrm{O}_{S}$ & 3558.66 & 0.2861 & 0.0 & $\mathrm{Gd}$ & 2.55 \\
$\mathrm{Mo}^{-} \mathrm{O}_{S}$ & 945.947 & 0.366617 & 0.0 & $\mathrm{Mo}$ & 5.1 \\
$\mathrm{O}_{S}-\mathrm{O}_{S}$ & 598.8379 & 0.314838 & 26.8965 & $\mathrm{O}_{C}$ & 0.746527 \\
$\mathrm{O}_{S}-\mathrm{O}_{C}$ & \multicolumn{2}{|c|}{$\chi=56.5628 \mathrm{eV} / \AA^{2}$} & $\mathrm{O}_{S}$ & -2.446527
\end{tabular}

упругим свойствам родственного кристалла $\mathrm{CaMoO}_{4}$ и целого ряда простых и сложных оксидов. Авторы работы [35] продемонстрировали трансферабельность полученных параметров при моделировании твердых растворов $\mathrm{NaEuMo}_{2} \mathrm{O}_{8}$ и $\mathrm{Ca}_{2} \mathrm{Mo}_{2} \mathrm{O}_{8}-\mathrm{NaEuMo}_{2} \mathrm{O}_{8}$. Поэтому при моделировании $\mathrm{Gd}_{2}\left(\mathrm{MoO}_{4}\right)_{3}$ были использованы параметры потенциалов молибден-кислород и кислород-кислород из работы [35].

Эмпирические параметры потенциалов межатомного взаимодействия гадолиний-кислород были определены нами путем процедуры подгонки к экспериментальным значениям параметров элементарной ячейки, координат атомов и упругих констант $\beta^{\prime}$-GMO, предусмотренной в рамках программы GULP. В качестве реперных значений для атомистического моделирования в настоящей работе использованы координаты атомов из работы [9], полученные на монокристаллических образцах.

Термодинамические функции, включая температурные зависимости теплоемкости при постоянном объеме и колебательной энтропии, были определены в гармоническом приближении из фононных спектров.

\section{3. Результаты и их обсуждение}

Параметры потенциалов гадолиний-кислород были получены в настоящей работе в процессе оптимизации структурных данных и упругих констант ромбической модификации $\mathrm{Gd}_{2}\left(\mathrm{MoO}_{4}\right)_{3}$ в соответствии с экспериментальными данными работ [9] и [36]. Все параметры потенциалов межатомных взаимодействий, полученные и использованные в работе, представлены в табл. 1. В табл. 2 приведены результаты оптимизации координат атомов в структуре $\beta^{\prime}$-GMO и сравнение их с реперными значениями из работы [9]. Видно их неплохое соответствие, что свидетельствует об адекватном выборе параметров потенциалов межатомных взаимодействий.

Таблица 2. Сравнение значений координат атомов в ромбических кристаллах $\mathrm{Gd}_{2}\left(\mathrm{MoO}_{4}\right)_{3}$ (пространственная группа $P b a 2$ ), полученных экспериментально [9] и после оптимизации параметров взаимодействия в настоящей работе

\begin{tabular}{|c|c|c|c|c|c|}
\hline Параметр & Эксперимент [9] & Расчет & Параметр & Эксперимент [9] & Расчет \\
\hline $\operatorname{Gd}(1) x$ & 0.1878 & 0.1953 & $\mathrm{O}_{c}(4) z$ & 0.690 & 0.688 \\
\hline $\operatorname{Gd}(1) y$ & 0.4954 & 0.5006 & $\mathrm{O}_{c}(5) x$ & 0.158 & 0.149 \\
\hline $\operatorname{Gd}(1) z$ & 0.7377 & 0.7377 & $\mathrm{O}_{c}(5) y$ & 0.156 & 0.153 \\
\hline $\operatorname{Gd}(2) x$ & 0.4930 & 0.4984 & $\mathrm{O}_{c}(5) z$ & 0.682 & 0.685 \\
\hline $\operatorname{Gd}(2) y$ & 0.3123 & 0.3050 & $\mathrm{O}_{c}(6) x$ & 0.157 & 0.154 \\
\hline $\operatorname{Gd}(2) z$ & 0.2632 & 0.2637 & $\mathrm{O}_{c}(6) y$ & 0.336 & 0.346 \\
\hline $\operatorname{Mo}(1) x$ & 0.2057 & 0.2078 & $\mathrm{O}_{c}(6) z$ & 0.307 & 0.307 \\
\hline $\operatorname{Mo}(1) y$ & 0.4890 & 0.4933 & $\mathrm{O}_{c}(7) x$ & 0.384 & 0.378 \\
\hline $\operatorname{Mo}(1) z$ & 0.3573 & 0.3602 & $\mathrm{O}_{c}(7) y$ & 0.384 & 0.375 \\
\hline $\operatorname{Mo}(2) x$ & 0.0029 & 0.0005 & $\mathrm{O}_{c}(7) z$ & 0.719 & 0.712 \\
\hline $\operatorname{Mo}(2) y$ & 0.2065 & 0.2086 & $\mathrm{O}_{c}(8) x$ & 0.385 & 0.376 \\
\hline $\operatorname{Mo}(2) z$ & 0.6426 & 0.6409 & $\mathrm{O}_{c}(8) y$ & 0.114 & 0.119 \\
\hline $\operatorname{Mo}(3) x$ & 0.2423 & 0.2472 & $\mathrm{O}_{c}(8) z$ & 0.294 & 0.296 \\
\hline $\operatorname{Mo}(3) y$ & 0.2428 & 0.2499 & $\mathrm{O}_{c}(9) x$ & 0.126 & 0.127 \\
\hline Mo $(3) z$ & 0 & 0.0005 & $\mathrm{O}_{c}(9) y$ & 0.171 & 0.185 \\
\hline $\mathrm{O}_{c}(1) x$ & 0.192 & 0.197 & $\mathrm{O}_{c}(9) z$ & 0.094 & 0.093 \\
\hline $\mathrm{O}_{c}(1) y$ & 0.488 & 0.494 & $\mathrm{O}_{c}(10) x$ & 0.317 & 0.312 \\
\hline $\mathrm{O}_{c}(1) z$ & 0.519 & 0.523 & $\mathrm{O}_{c}(10) y$ & 0.126 & 0.131 \\
\hline $\mathrm{O}_{c}(2) x$ & 0.480 & 0.483 & $\mathrm{O}_{c}(10) z$ & 0.907 & 0.906 \\
\hline $\mathrm{O}_{c}(2) y$ & 0.305 & 0.301 & $\mathrm{O}_{c}(11) x$ & 0.354 & 0.363 \\
\hline $\mathrm{O}_{c}(2) z$ & 0.482 & 0.478 & $\mathrm{O}_{c}(11) y$ & 0.320 & 0.315 \\
\hline $\mathrm{O}_{c}(3) x$ & 0.129 & 0.129 & $\mathrm{O}_{c}(11) z$ & 0.098 & 0.098 \\
\hline $\mathrm{O}_{c}(3) y$ & 0.007 & 0.005 & $\mathrm{O}_{c}(12) x$ & 0.170 & 0.182 \\
\hline $\mathrm{O}_{c}(3) z$ & 0.311 & 0.314 & $\mathrm{O}_{c}(12) y$ & 0.357 & 0.367 \\
\hline $\mathrm{O}_{c}(4) x$ & 0.494 & 0.494 & $\mathrm{O}_{c}(12) z$ & 0.902 & 0.904 \\
\hline $\mathrm{O}_{c}(4) y$ & 0.128 & 0.129 & & & \\
\hline
\end{tabular}


В табл. 3 показаны результаты расчета параметров элементарной ячейки, ее объема, а также упругих констант в сравнении с экспериментальными данными. Значения параметров элементарной ячейки $a$ и $b$ воспроизводят экспериментальные данные с точностью до сотых долей процента. Для параметра $c$, совпадающего с направлением поляризации сегнетоэлектрика, расчетное значение на 2.6\% меньше экспериментального, для объема элементарной ячейки отличие составляет $1.7 \%$.

Расчетные значения упругих констант в большинстве случаев несколько больше экспериментальных, однако эти различия невелики и сопоставимы с разбросом экспериментальных данных, полученных разными авторами.

Таким образом, модель с используемым набором потенциалов взаимодействия и эффективными значениями зарядов атомов позволяет неплохо описать структурные и упругие свойства кристаллов $\beta^{\prime}$-GMO.

В результате моделирования был рассчитан также целый ряд физических и термодинамических характеристик ромбической модификации $\mathrm{Gd}_{2}\left(\mathrm{MoO}_{4}\right)_{3}$. Данные о температурной зависимости теплоемкости представлены на рис. 2. Для сопоставления с экспериментальными данными значения теплоемкости при постоянном объеме $C_{V}$, получаемые в процессе моделирования, пересчитывались в значения $C_{p}$ (теплоемкости при постоянном давлении), измеряемые экспериментально. Для этого использовалось соотношение [41]

$$
C_{p}=C_{V}+\alpha^{2} T V / \beta
$$

где $\alpha-$ коэффициент объемного расширения, $T$ - температура, $V$ - мольный объем, $\beta$ - сжимаемость. Необходимые для расчета величины были получены с использованием экспериментальных данных работ [42,36,43].

Таблица 3. Сравнение структурных параметров и упругих констант ромбической $\beta^{\prime}$-модификации $\mathrm{Gd}_{2}\left(\mathrm{MoO}_{4}\right)_{3}$ по результатам моделирования и эксперимента

\begin{tabular}{c|c|c|c|c}
\hline Свойства & Расчет & \multicolumn{3}{|c}{ Эксперимент } \\
\hline$a, \AA$ & 10.3928 & $10.3881[9]$ & $10.40[1]$ & $10.3858[3]$ \\
$b, \AA$ & 10.4172 & $10.4194[9]$ & $10.40[1]$ & $10.4186[3]$ \\
$c, \AA$ & 10.4194 & $10.7007[9]$ & $10.66[1]$ & $10.7003[3]$ \\
$V, \AA^{3}$ & 1137.51 & $1158.22[9]$ & - & $1157.844[3]$ \\
$c_{i j}, \cdot 10^{10}$ & & {$[36]$} & {$[37]$} & {$[38]$} \\
$\mathrm{N} / \mathrm{m}^{2}$ & & & & \\
$c_{11}$ & 8.15 & 5.85 & 6.95 & 7.60 \\
$c_{22}$ & 9.25 & 7.30 & 6.95 & 7.60 \\
$c_{33}$ & 11.05 & 10.30 & 9.95 & 9.70 \\
$c_{44}$ & 3.22 & 2.45 & 2.55 & 2.38 \\
$c_{55}$ & 3.20 & 2.60 & 2.55 & 2.92 \\
$c_{66}$ & 2.28 & 3.30 & 2.50 & - \\
$c_{12}$ & 2.22 & 1.05 & - & - \\
$c_{13}$ & 3.87 & 2.85 & - & - \\
$c_{23}$ & 3.99 & 3.20 & - & -
\end{tabular}

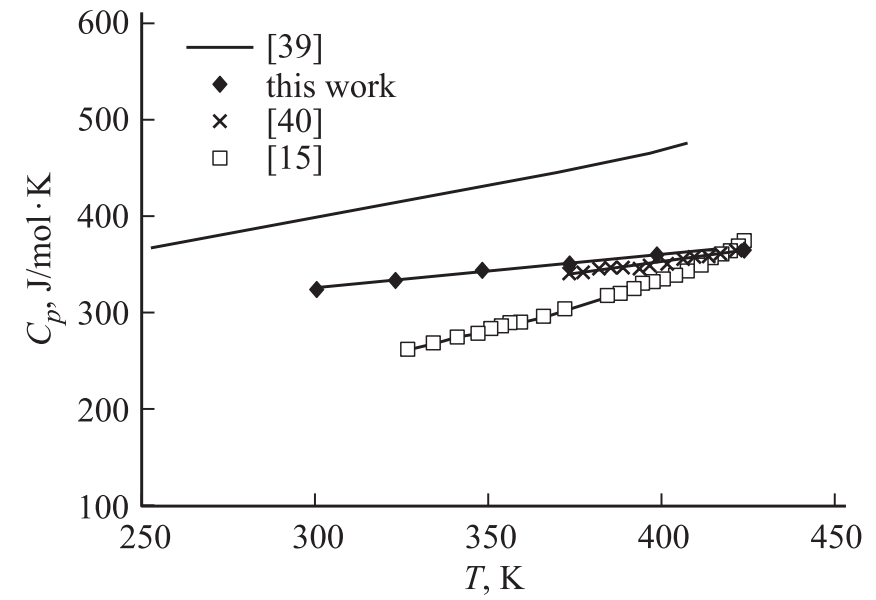

Рис. 2. Зависимость теплоемкости при постоянном давлении от температуры по результатам расчета (настоящая работа) и экспериментальным данным (работы $[15,39,40]$ ).

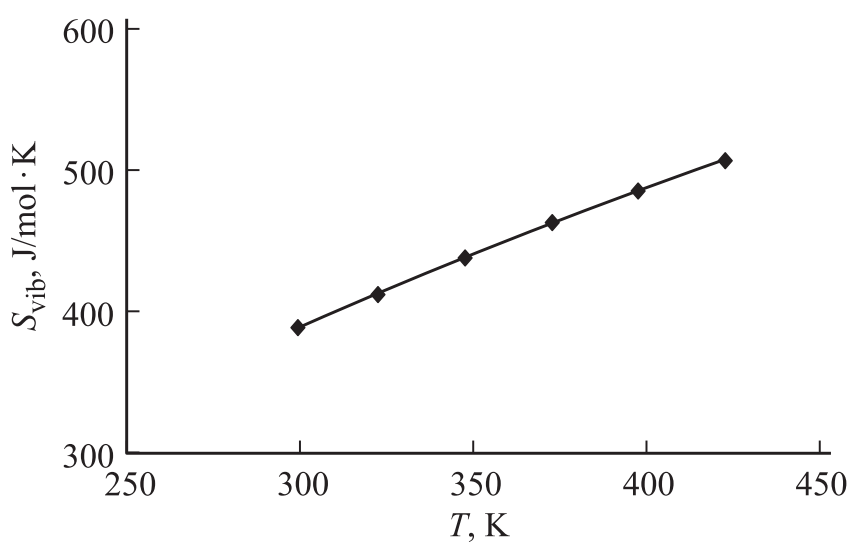

Рис. 3. Температурная зависимость колебательной энтропии по результатам моделирования.

Экспериментальные данные по температурным зависимостям теплоемкости $[15,39,40]$ дают согласующиеся результаты относительно температуры фазового перехода (на рис. 2 не показан). Однако теплоемкость $\beta^{\prime}$-фазы, которая является предметом исследования настоящей работы, по данным разных авторов заметно различается. Различия между экспериментальными значениями достигают 40\%. Наши расчетные значения лежат между экспериментальными результатами работ [15] и [39] и хорошо согласуются с экспериментальными данными работы [40].

По результатам наших вычислений в рассмотренном интервале температур зависимость теплоемкости от температуры близка к линейной и может быть описана уравнением

$$
C_{p}=0.3346 T+225.72 \mathrm{~J} /(\mathrm{mol} . \mathrm{K})
$$

с коэффициентом корреляции $R^{2}=0.9928$.

Более точной является зависимость

$$
C_{p}=-0.0008 T^{2}+0.9105 T+123.08
$$

(при $\left.R^{2}=1\right)$. 
Таблица 4. Характеристики кристаллов ромбической $\beta^{\prime}$-модификации $\mathrm{Gd}_{2}\left(\mathrm{MoO}_{4}\right)_{3}$, полученные по результатам моделирования и по экспериментальным данным

\begin{tabular}{c|c|c}
\hline Характеристики & Моделирование & Эксперимент \\
\hline$\rho, \mathrm{g} / \mathrm{cm}^{3}$ & 4.68 & $4.56[43]$ \\
& & $4.565[38]$ \\
$K, \mathrm{GPa}$ & 54.04 & $41.8^{a}$ \\
$G, \mathrm{GPa}$ & 29.65 & $27.6^{a}$ \\
$E, \mathrm{GPa}$ & 67.14 & $62.5^{b}$ \\
$\beta, \mathrm{GPa}$ & $0.0239^{a}$ \\
$V_{s}, \mathrm{~km} / \mathrm{s}$ & 0.0193 & $2.40[44]$ \\
& 2.51 & $2.36[37]$ \\
$V_{p}, \mathrm{~km} / \mathrm{s}$ & 4.47 & $4.65[44]$ \\
& & $3.9[37]$ \\
$E_{s t r}, \mathrm{eV}$ & 598.69 & - \\
$C_{V},(300 \mathrm{~K})$ & 320 & - \\
$\mathrm{J} /(\mathrm{mol} . \mathrm{K})$ & & $341[40]$ \\
$C_{p},(373 \mathrm{~K})$ & 351 & $299[15]$ \\
$\mathrm{J} /(\mathrm{mol} . \mathrm{K})$ & & - \\
$S_{v i b},(300 \mathrm{~K})$ & 390 & \\
$\mathrm{~J} /(\mathrm{mol} . \mathrm{K})$ & &
\end{tabular}

Примечание. Значками $a$ и $b$ отмечены оценки, сделанные нами при использовании экспериментальных данных из работ [36] и [45] соответственно.

Зависимость колебательной энтропии от температуры представлена на рис. 3. Эта зависимость в рассмотренном температурном интервале также близка к линейной и может быть описана уравнением

$$
S_{v i b}=0.951 T+106.59
$$

(при $\left.R^{2}=0.9989\right)$. Более точно зависимость колебательной энтропии от температуры описывается полиномом второй степени

$$
S_{v i b}=-0.0009 T^{2}+1.5861 T-6.5992
$$

(при $R^{2}=1$ ). Численные значения теплоемкости для 300 и $373 \mathrm{~K}$, а также колебательной энтропии для $300 \mathrm{~K}$ представлены в табл. 4.

В табл. 4 приведены также оценки таких свойств моделируемых кристаллов, как плотность $\rho$, модуль объемной упругости $K$, модуль сдвига $G$, модуль Юнга $E$, сжимаемость $\beta$, скорости продольных $V_{p}$ и поперечных $V_{s}$ акустических волн, структурная энергия $E_{s t r}$. Для механических свойств приведены значения, рассчитанные по усреднению Фойгта [46]. Сравнение результатов моделирования с экспериментальными данными показывает их неплохое соответствие. Это свидетельствует об удачном выборе параметров потенциалов межатомного взаимодействия, используемых в работе. Наши предварительные результаты моделирования двойного молибдата $\mathrm{NaGdMo}_{2} \mathrm{O}_{8}$ подтверждают трансферабельность этих параметров на более сложные системы.

\section{4. Заключение}

Проведено атомистическое моделирование кристалла молибдата гадолиния с использованием системы потенциалов межатомного взаимодействия и эффективных значений зарядов атомов. Параметры потенциалов межатомного взаимодействия $\mathrm{Gd}-\mathrm{O}$ получены в процессе оптимизации структурных данных и упругих констант $\beta^{\prime}$-GMO.

Рассчитаны важнейшие физические, механические и термодинамические характеристики $\beta^{\prime}$-GMO, такие как плотность, модуль объемной упругости, модуль сдвига, модуль Юнга, сжимаемость, скорости продольных и поперечных акустических волн, структурная энергия, а также получены температурные зависимости теплоемкости и колебательной энтропии. Сравнение результатов расчета с экспериментальными данными свидетельствует об адекватности использованной модели для описания сегнетоэлектрика-сегнетоэластика $\mathrm{Gd}_{2}\left(\mathrm{MoO}_{4}\right)_{3}$.

\section{Список литературы}

[1] H.J. Borchardt, P.E. Bierstedt. Appl. Phys. Lett. 8, 50 (1966).

[2] C.T. Prewitt. Solid State Commun. 8, 2037 (1970).

[3] E.T. Keve, S.C. Abrahams, J.L. Bernstein. J. Chem. Phys. 54, 3185 (1971).

[4] Q. Yuan, C. Zhao, W. Luo, X. Yin, J. Xu, S. Pan. J. Cryst. Growth. 233, 717 (2001).

[5] B. Joukoff, G. Grimouille. J. Cryst. Growth. 43, 719 (1978).

[6] E.T. Keve, S.C. Abrahams, K. Nassau, A.M. Glass. Solid State Commun. 8, 1517 (1970).

[7] K. Nassau, J.W. Shiever, E.T. Keve. J. Solid State Chem. 3, 411 (1971).

[8] W. Jeitschko. Naturwiss. 27, 544 (1970).

[9] W. Jeitschko. Acta Cryst. B 28, 60 (1972).

[10] G. Lucazeau, D. Machon. J. Raman Spectrosc. 37, 189 (2006).

[11] G. Lucazeau, P. Bouvier, A. Pasturel, O. Le Bacq, T. Pagnier. Acta Phys. Polonica A, 116, 25 (2009).

[12] V.A. Morozov, M.V. Raskina, B.I. Lazoryak, K.W. Meert, K. Korthout, P.F. Smet, D. Poelman, N. Gauquelin, J. Verbeeck, A.M. Abakumov, J. Hadermann. Chem. Mater. 26, 7124 (2014).

[13] J.M. Perez-Mato, D. Orobengoa, M.I. Aroyo. Acta Cryst. A 66, 558 (2010).

[14] V. Dvorak. Phys. Status Solidi. B 45, 147 (1971).

[15] K.M. Cheung, F.G. Ullman. Phys. Rev. B 10, 4760 (1974).

[16] D. Jaque, Z.D. Luo, J.G. Sole. Appl. Phys. B 72, 811 (2001).

[17] J. Tang, Y. Chen, Y. Lin, X. Gong, J. Huang, Z. Luo, Y. Huang. Opt. Express 19, 13185 (2011).

[18] J. Tang, Y. Chen, Y. Lin, Y. Huang. J. Lumin. 138, 15 (2013).

[19] L.L. Yang, J.F. Tang, J.H. Huang, X.H. Gong, Y.J. Chen, Y.F. Lin, Z.D. Luo, Y.D. Huang. Opt. Mater. 35, 2188 (2013).

[20] С.3. Шмурак, А.П. Киселев, Д.М. Курмашева, Б.С. Редькин, В.В. Синицын. ЖЭТФ 137, 867 (2010).

[21] V.V. Sinitsyn, B.S. Redkin, A.P. Kiselev, S.Z. Shmurak, N.N. Kolesnikov, V.V. Kveder, E.G. Ponyatovsky. Solid State Sci. 46, 80 (2015).

[22] Y.X. Pan, Q.Y. Zhang. Mater. Sci. Eng. B 138, 90 (2007).

[23] D.P. Dutta, A.K. Tyagi. Solid State Phenomena 155, 113 (2009). 
[24] Y. Wang, T. Honma, Y. Doi, Y. Hinatsu, T. Komatsu. J. Ceram. Soc. Jpn 121, 230 (2013).

[25] L. Bufaiçal, G. Barros, L. Holanda, I. Guedes. J. Magn. Magn. Mater. 378, 50 (2015).

[26] D. Jaque, J. Findensein, E. Montoya, J. Capmany, A.A. Kaminskii, H.J. Eichler, J.G. Sole. J. Phys.: Condens. Matter 12, 9699 (2000).

[27] M. Li, S. Sun, L. Zhang, Y. Huang, F. Yuan, Z. Lin. Opt. Commun. 355, 89 (2015).

[28] Акустические кристаллы. Справочник / Под ред. М.П. Шаскольской. Наука, М. (1982). 632 с.

[29] D.N. Nikogosyan. Nonlinear Optical Crystals: A Complete Survey. Springer, N. Y. (2005). 429 p.

[30] В.С. Урусов, В.Б. Дудникова. Геохимия 49, 1097 (2011).

[31] J.D. Gale. Zeitschrift für Kristallographie 220, 552 (2005).

[32] B.G. Dick, A.W. Overhauser. Phys. Rev. 112, 90 (1958).

[33] K. Leinenweber, A. Navrotsky. Phys. Chem. Minerals 15, 588 (1988).

[34] C.I. Sainz-Diaz, A. Hernandez-Laguna, M.T. Dove. Phys. Chem. Minerals 28, 130 (2001).

[35] V.L. Vinograd, D. Bosbach, B. Winkler, J.D. Gale. Phys. Chem. Chem. Phys. 10, 3509 (2008).

[36] M. Busch, J.C. Toledano, J. Torres. Opt. Commun. 10, 273 (1974).

[37] D.J. Epstein, W.V. Herrick, R. F. Turek. Solid State Commun. 8, 1491 (1970).

[38] S. Mielcarek, A. Trzaskowska, B. Mroz, T. Andrews. J. Phys.: Condens. Matter. 17, 587 (2005).

[39] B. Strukov, I. Shnaidshtein, A. Onodera. Ferroelectrics 363, 27 (2008).

[40] A. Fouskova. J. Phys. Soc. Jpn 27, 1699 (1969).

[41] Р.А. Свелин. Термодинамика твердого состояния. Металлургия, М. (1968). 316 с.

[42] J. Kobayashi, Y. Sato, T. Nakamura. Phys. Status Solidi. A 14, 259 (1972).

[43] J. Sapriel, R. Vacher. J. Appl. Phys. 48, 1191 (1977).

[44] И.А. Андреев. Изв. РГПУ им. А.И. Герцена. 6, 27 (2006).

[45] T. Nakamura, E. Sawaguchi. J. Phys. Soc. Jpn 50, 2323 (1981).

[46] Дж. Най. Физические свойства кристаллов и их описание при помощи тензоров и матриц. Мир, М. (1967). 386 с. 